\title{
Cellular swirls in fine needle aspirates of papillary thyroid carcinoma: a new diagnostic criterion
}

\author{
Arnold H Szporn, Songyang Yuan, Maoxin Wu and David E Burstein \\ Division of Cytopathology, Department of Pathology, Mount Sinai School of Medicine, New York, NY, USA
}

\begin{abstract}
No single cytologic feature is specifically diagnostic for papillary thyroid carcinoma. We report herein the presence of swirl-like cellular aggregates in fine needle aspirates of papillary thyroid carcinoma but not in other thyroid entities. Cellular swirls are defined as concentrically organized aggregates of tumor cells in which many of the most peripherally situated cells have ovoid rather than round nuclei that are oriented perpendicular to the radius of the swirl. One hundred Papanicolaou- and/or Diff-Quik-stained FNAs of the thyroid diagnosed as papillary carcinoma, including seven fine needle aspirates of cervical lymph nodes showing metastatic papillary carcinoma, with or without cell blocks, were reviewed for the presence of cellular swirls. An additional 100 thyroid FNAs, similarly stained and prepared, diagnosed as nodular goiter, Hashimoto's thyroiditis and follicular neoplasm were also reviewed for the presence of cellular swirls. Cellular swirls were easily observed at screening magnification and confirmed at high magnification. Seventeen of 100 FNAs $(17 \%)$ of papillary carcinoma contained cellular swirls. No cases diagnosed as nodular goiter, Hashimoto's thyroiditis or follicular neoplasm contained these structures. Thirteen cases with swirls had histologic follow-up. These comprised seven papillary carcinomas with classical histopathology, two designated 'differentiated papillary carcinoma,' two with follicular variant histopathology; one with a minor component of follicular variant histopathology; one papillary carcinoma metastatic to a cervical lymph node with classic histopathology. Swirls occurred in cases with relatively little pleomorphism, or in well-differentiated regions of papillary carcinoma that also displayed less well-differentiated components. Cellular swirls are a finding that is highly specific to papillary thyroid carcinoma. They are easily seen at screening magnification. Their presence in a FNA specimen may be helpful in cases where classic criteria for papillary thyroid carcinoma are scarce, particularly in well-differentiated papillary thyroid carcinoma. While the size and scope of this study are insufficient to conclude that cellular swirls alone are diagnostic of papillary thyroid carcinoma in the absence of other criteria, we believe these structures should be added to the list of diagnostic criteria.
\end{abstract}

Modern Pathology (2006) 19, 1470-1473. doi:10.1038/modpathol.3800669; published online 1 September 2006

Keywords: cellular swirls; papillary thyroid carcinoma; fine needle aspiration; cytology; thyroid

Thyroid carcinoma is the most common endocrine malignancy, accounting for approximately $1 \%$ of all malignant diagnoses. ${ }^{1}$ Papillary thyroid carcinoma (PTC) accounts for $70-80 \%$ of thyroid cancers. ${ }^{1}$ Fine needle aspirates (FNAs) are an important modality for establishing the pre-surgical diagnosis of PTC. ${ }^{2-5}$ No single cytologic feature is specifically diagnostic for PTC; the finding of several recognized criteria make the diagnosis of PTC more likely in any given case. We describe herein a new criterion for the diagnosis of PTC on FNA, the presence of cell

Correspondence: Dr DE Burstein, MD, Department of Pathology, Box 1194, Mount Sinai School of Medicine, 1 Gustave L. Levy Place, New York, NY 10029, USA.

E-mail: david.burstein@mssm.edu

Received 17 January 2006; revised and accepted 3 July 2006; published online 1 September 2006 aggregates that we refer to as cellular swirls. As described below, these structures were present in FNAs of PTC, but not in FNAs of other thyroid entities. Cellular swirls are defined as flat, concentrically organized aggregates of about 50-200 tumor cells, in which many of the most peripherally situated cells have ovoid rather than round nuclei that are oriented perpendicular to the radius of the swirl. To our knowledge, these structures have not been previously reported in the literature and in particular, their relationship to PTC is undescribed.

\section{Materials and methods}

One hundred Papanicolaou-stained and/or DiffQuik-stained smears comprising 93 FNAs of the 
thyroid diagnosed as PTC and seven FNAs from cervical lymph nodes with metastatic PTC, with or without cell blocks, were reviewed for the presence of cellular swirls. An additional 100 similarly stained and prepared thyroid FNAs, diagnosed as nodular goiter, Hashimoto's thyroiditis or follicular neoplasm, were also reviewed for the presence of cellular swirls. In addition, the presence of structures with similarities to, but which do not strictly match our definition of cellular swirls, was also recorded. All 100 cases of PTC had either confirmatory surgical pathology follow-up $(n=49)$ or were diagnostic on cytologic grounds, based upon the presence of multiple classic criteria for diagnosing PTC, including intranuclear inclusions and grooves, monolayered sheets, giant cells, papillary structures and psammoma bodies. Seven of 100 non-PTC FNA cases had histological follow-up. Of these, three were follicular adenomas, one follicular cell neoplasm with extensive oncocytic differentiation, one multinodular goiter, one adenomatous nodule/ follicular neoplasm cannot be ruled out and one Hashimoto's thyroiditis.

\section{Results}

Cellular swirls were easily observed at screening magnification and confirmed at high magnification (Figure 1). These structures were relatively flat rather than ball-like and did not contain any colloid. Cellular swirls consisted of concentrically organized aggregates of about 50-200 tumor cells, in which most of the peripherally situated cells have ovoid rather than round nuclei, the long axes of which were oriented perpendicular to the radius of the swirl (Figure 2). Often, cellular swirls were contiguous with flat sheets of tumor cells with similar cytologic features. The nuclear chromatin in most cases was bland and uniform and nuclear membranes were smooth and unremarkable. Occasional cells showed a nuclear groove or a small nucleolus. Nuclear pleomorphism was not present within the swirls. The cytoplasm was usually scant and delicate and cytoplasmic borders indistinct. However, several swirls were composed of cells with relatively dense cytoplasm and distinct cytoplasmic borders (Figure 3). Cellular swirls were not directly associated with psammoma bodies or multinucleated giant cells. Seventeen of 100 FNAs $(17.0 \%)$ of PTCs contained cellular swirls, 16 in thyroid FNAs and one in a cervical lymph node FNA. The number of swirls varied from one to three per case. Cellular swirls were found in seven papillary carcinomas with classical histopathology, two designated 'differentiated papillary carcinoma,' two with follicular variant histopathology and one with a minor component of follicular variant histopathology. In addition, a single metastatic PTC with classical histopathology in a cervical lymph node demonstrated a swirl. Examination of

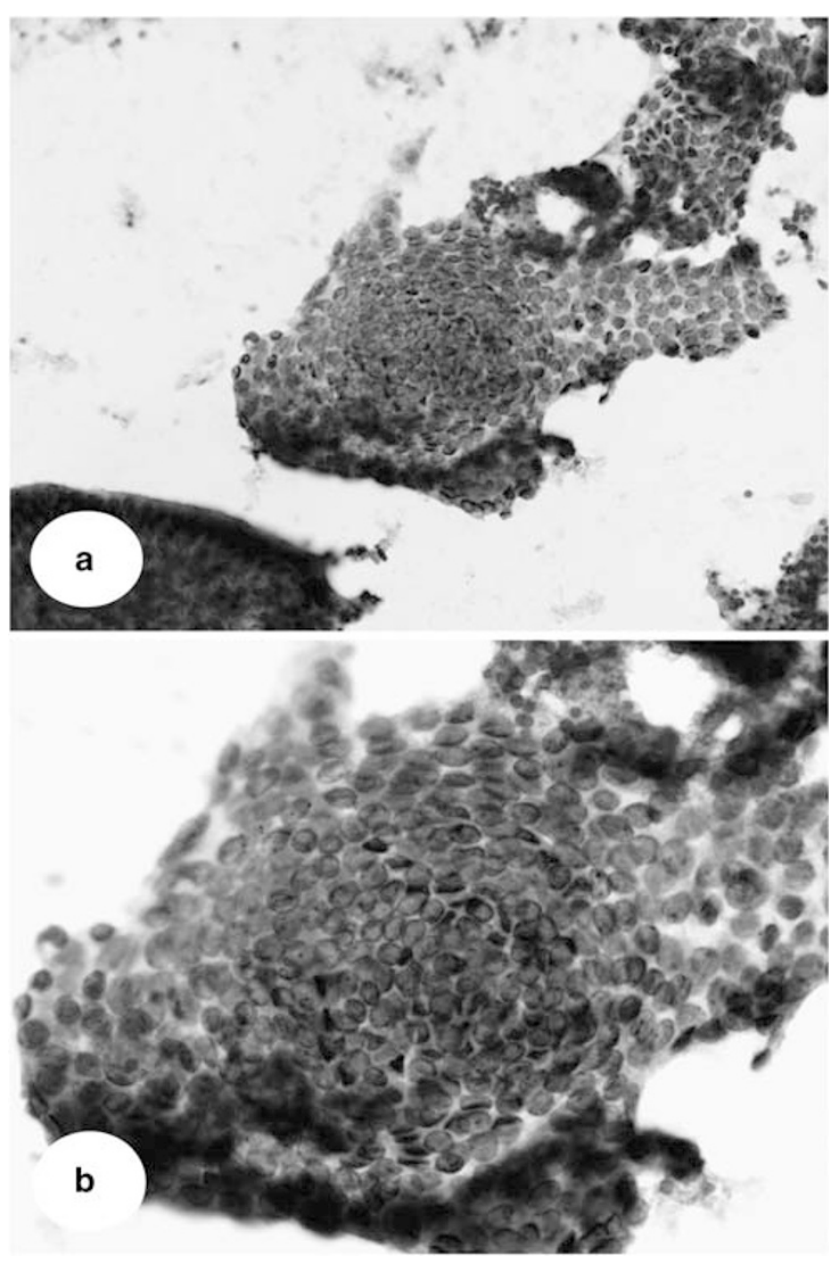

Figure 1 (a) Cellular swirl at screening magnification (Papanicolaou, $\times 20$ ). (b) Cellular swirl at high magnification (Papanicolaou, $\times 40)$.

available histopathologic sections in a few cases with swirls on FNA, showed no structure resembling cellular swirls. No cases diagnosed as nodular goiter, Hashimoto's thyroiditis or follicular neoplasm contained these structures. Structures similar to, but not completely morphologically characteristic of cellular swirls were also observed in seven cases of PTC (7\%) (Figure 4). They differed from true cellular swirls in that their nuclei were round rather than ovoid, or architecturally, had variable degrees of disoriented and/or incompletely concentric nuclear long axes. These partially swirl-like structures were not found in any non-PTC cases.

\section{Discussion}

In 1980, Kini et al ${ }^{6}$ suggested that diagnostic criteria for PTC include papillary tissue fragments, monolayered sheets, syncytial tissue fragments, intranuclear cytoplasmic inclusions, psammoma bodies and multinucleated giant cells. 'Ropy' colloid was first described by Löwhagen. ${ }^{7}$ Criteria described by 


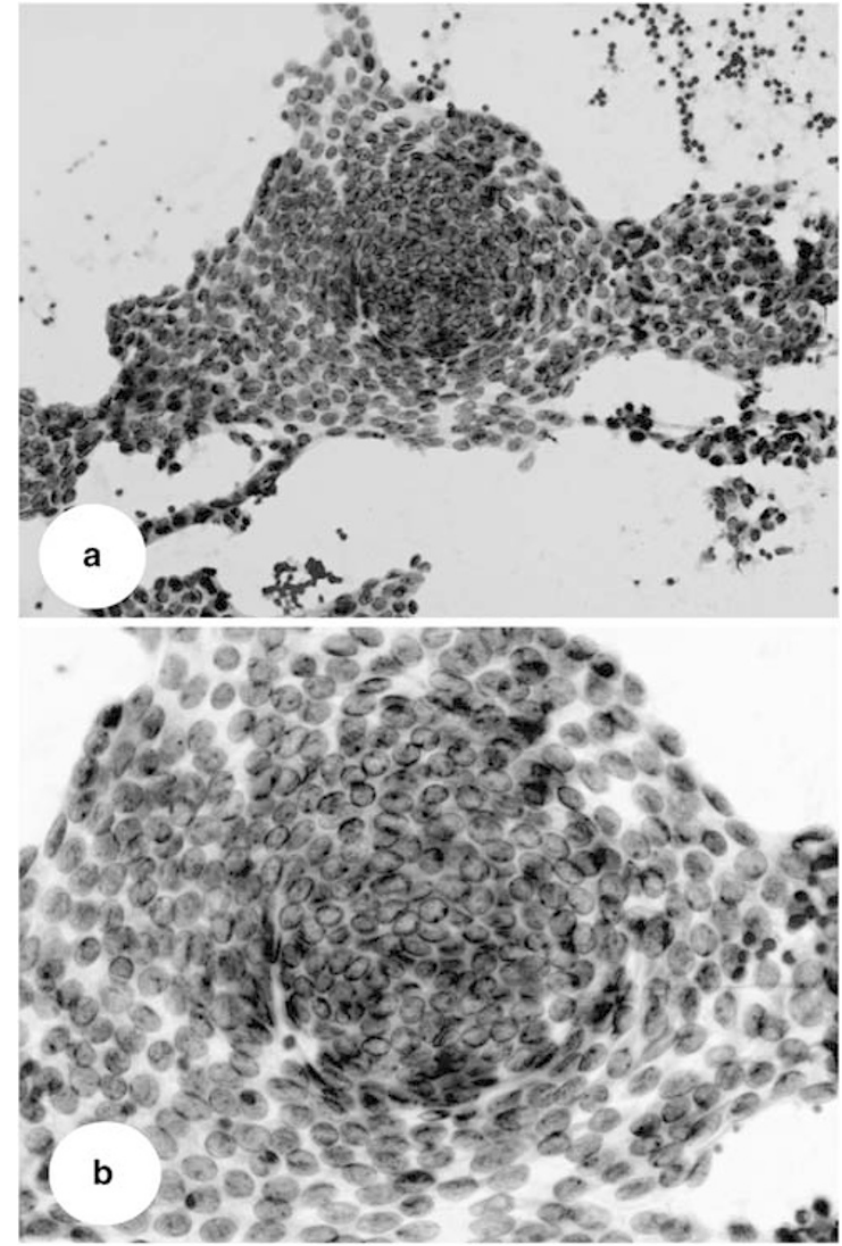

Figure 2 (a) Cellular swirl contiguous with flat sheet of tumor cells with similar cytologic features (Papanicolaou, $\times 20$ ). $($ b) Cellular swirl showing concentrically organized aggregate of tumor cells with indistinct cytoplasmic borders with the most peripherally situated nuclei being ovoid rather than round and their long axes perpendicular to the radius of the swirl (Papanicolaou, $\times 40)$.

Koss included cellular rich aspirates with little colloid, sharply outlined papillary clusters, distinct cytoplasmic borders, enlarged nuclei, nucleoli, intranuclear cytoplasmic inclusions and psammoma bodies. ${ }^{8}$ Although nuclear grooves were described by Chan and Shaw in histological sections and imprint smears of PTC, ${ }^{9}$ their utility as a diagnostic feature in FNAs was first reported by Rupp and Ehya. ${ }^{10}$ The list of criteria for the diagnosis of PTC by FNA has continued to grow. More recently, DeMay recognized 13 criteria divided into four categories, including architectural (3-dimensional papillae with cores, 3-dimensional caps without cores, 2-dimensional sheets with fingers), cytoplasmic (squamoid features, septate vacuoles), nuclear (grooves, intranuclear cytoplasmic inclusions, fine pale chromatin, marginated nucleoli) and background (psammoma bodies, epithelioid giant cells, gummy colloid). ${ }^{11}$
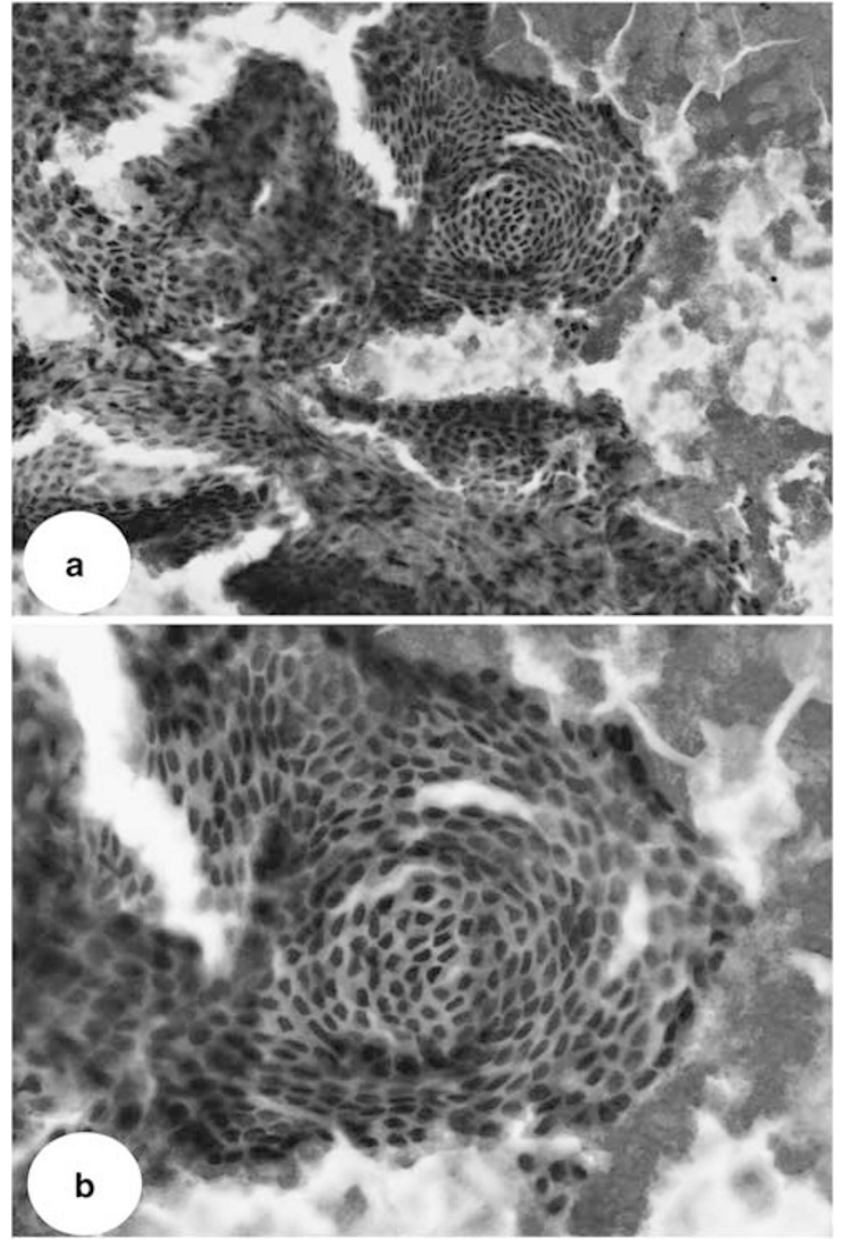

Figure 3 (a) Cellular swirl displaying distinct cell borders and relatively dense cytoplasm at screening magnification (Papanicolaou, $\times 20)($ b) Cellular swirl at high magnification (Papanicolaou, $\times 40$ ).

The findings described herein suggest the existence of a new criterion, the cellular swirl. Cellular swirls are found in a significant fraction of FNAs of PTC, but not FNAs of other thyroid lesions. They are observed in association with relatively well-differentiated tumor cells in sheets and are easily seen at screening magnification.

Cellular swirls should not be confused with the spherules (3-dimensional balls of follicular cells with or without colloid content) that may be seen in thyroid FNAs. ${ }^{12,13}$ Mesonero et $a 1^{13}$ associated such spherules with the macrofollicular subtype of the follicular variant of PTC. In contrast, cellular swirls are relatively flat, 2-dimensional structures and are not associated with colloid. Kuma et $a l^{14}$ reported whorl-like structures consisting of cells with mildly enlarged nuclei, thickened nuclear membranes and 'entirely clear contents' in a thyroid FNA of the cribriform-morular variant of PTC. These structures, the cells of which have prominent nuclear changes, show no similarities to the cellular swirls described presently, in which bland nuclear features are found. 


\section{Acknowledgement}

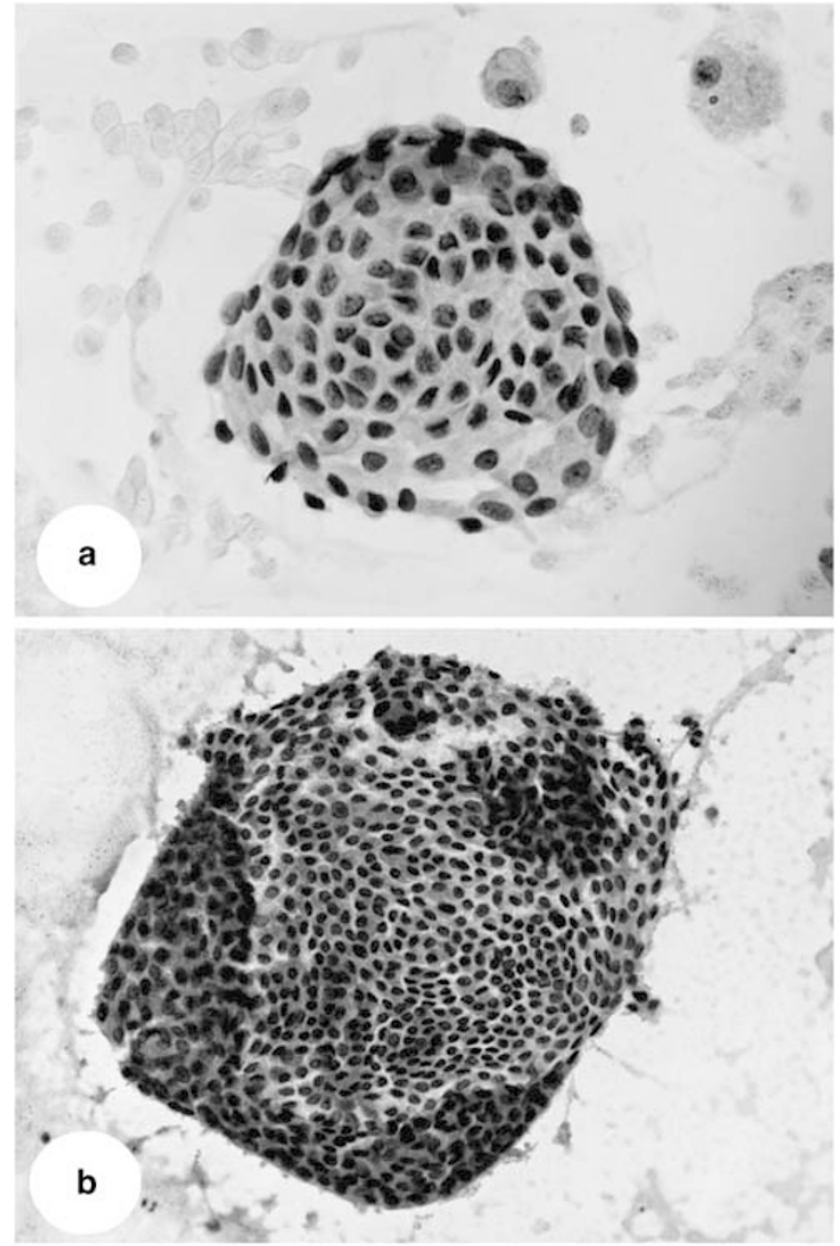

Figure 4 (a) Swirl-like structure with round rather than ovoid nuclei (Papanicolaou, $\times 40)($ b) Swirl-like structure with focally disoriented and incompletely concentric nuclear axes (Papanicolaou, $\times 20$ ).

It is well recognized that no single classic diagnostic feature of PTC in FNAs is present in all cases. Psammoma bodies, for example, are seen in only $20-40 \%$ of PTCs. ${ }^{11}$ More recently reported features associated with PTC FNAs include histiocytoid cells, seen in up to $6 \%$ of FNAs of PTC ${ }^{15}$ and dark and pale cerebriform nuclei, seen in up to $50 \%$ of the FNAs of PTCs reviewed. ${ }^{16}$ The presence of cellular swirls in $17 \%$ of our cases series falls between these two in prevalence.

We hypothesize that cellular swirls may represent areas of incipient papillation within flat sheets of tumor cells. They appear in most cases to be contiguous with sheets of tumor cells with similar cytologic features.

While the size and scope of this study are insufficient to conclude that cellular swirls are uniquely specific to and therefore diagnostic of PTC in the absence of other criteria, we believe that these structures are highly specific to PTC and should be added to the list of diagnostic criteria currently in practice.
We thank Mr Joseph Samet for his invaluable assistance in figure preparation.

\section{References}

1 Busnardo B, DeVido D. The epidemiology and etiology of differentiated thyroid carcinoma. Biomed Pharmacother 2000;54:322-326.

2 El Hag IA, Kollur SM, Chiedozi LC. The role of FNA in the initial management of thyroid lesions: 7-year experience in a district general hospital. Cytopathol 2003;14:126-130.

3 Fernandes JK, Day TA, Richardson MS, et al. Overview of the management of differentiated thyroid cancer. Curr Treat Options Oncol 2005;6:47-57.

4 Gharib H. Changing trends in thyroid practice: understanding nodular thyroid disease. Endocr Pract 2004; 10:31-39.

5 Zagorianakou P, Malamou-Mitsi V, Zagoriananoku N, et al. The role of fine-needle aspiration biopsy in the management of patients with thyroid nodules. In Vivo 2005;19:605-609.

6 Kini SR, Miller JM, Hamburger JI, et al. Cytopathology of papillary carcinoma of the thyroid by fine needle aspiration. Acta Cytol 1980;24:511-521.

7 Lowhagen T, Willems JS, Lundell G, et al. Aspiration biopsy cytology in diagnosis of thyroid cancer. World J Surg 1981;5:61-73.

8 Koss LG, Woyke S, Olszewski W. Aspiration biopsy: cytologic interpretation and histologic bases. IgakuShoin Medical Publishers, Inc.: New York, 1984, pp 169-170.

9 Chan JKC, Shaw D. The grooved nucleus: a useful diagnostic criterion of papillary carcinoma of the thyroid. Am J Surg Pathol 1986;10:672-679.

10 Rupp M, Ehya H. Nuclear grooves in the aspiration cytology of papillary carcinoma of the thyroid. Acta Cytol 1989;33:21-26.

11 DeMay RM. The art and science of cytopathology. American Society of Clinical Pathologists: Chicago, 1996, pp 730-734.

12 Frost AR, Sidawy MK, Ferfelli M, et al. Utility of thinlayer preparations in thyroid fine-needle aspiration: diagnostic accuracy, cytomorphology and optimal sample preparation. Cancer Cytopathol 1998;84: $17-25$.

13 Mesonero CE, Jungle JE, Wilbur DC, et al. Fine-needle aspiration of the macrofollicular and microfollicular subtypes of the follicular variant of papillary carcinoma of the thyroid. Cancer Cytopathol 1998;84: 235-244.

14 Kuma S, Hirokawa M, Xu B, et al. Cribriform-morular variant of papillary thyroid carcinoma. Report of a case showing morules with peculiar nuclear clearing. Acta Cytol 2004;48:431-436.

15 Renshaw AA. 'Histiocytoid cells in fine-needle aspitarions of papillary carcinoma of the thyroid: frequency and significance of an under-recognized cytologic pattern. Cancer Cytopathol 2002;96:240-243.

16 Mallik MK, Das DK, Mallik AA, et al. Dark and pale cerebrifiorm nuclei in fna smears of usual papillary thyroid carcinoma and its variants. Diagn Cytopathol 2004;30:187-192. 